\title{
Pediatric Life After Concussion Evaluation Scale (PLACES): Utility of a Survey of Quality of Life During Recovery from Concussion
}

\author{
Ruta Clair ${ }^{1, *}$, Arlene Goodman ${ }^{2}$, George McCloskey $^{3}$, Sarah Levin Allen $^{4}$ \\ ${ }^{1}$ Department of Psychology, Cabrini University, Radnor, United States \\ ${ }^{2}$ Saint Peter's University Hospital Sports Medicine Institute, Somerset, United States \\ ${ }^{3}$ Department of Psychology, Philadelphia College of Osteopathic Medicine, Philadelphia, United States \\ ${ }^{4}$ Brain Behavior Bridge, Haddon Heights, United States
}

Email address:

ruta.dr.clair@cabrini.edu (R. Clair), agoodman@saintpetersuh.com (A. Goodman), GeorgeMcC@pcom.edu (G. McCloskey), drallen@brainbehaviorbridge.com (S. L. Allen)

${ }^{*}$ Corresponding author

\section{To cite this article:}

Ruta Clair, Arlene Goodman, George McCloskey, Sarah Levin Allen. Pediatric Life After Concussion Evaluation Scale (PLACES): Utility of a Survey of Quality of Life During Recovery from Concussion. International Journal of Psychological and Brain Sciences.

Vol. 4, No. 6, 2019, pp. 56-64. doi: 10.11648/j.ijpbs.20190406.11

Received: November 12, 2019; Accepted: December 2, 2019; Published: December 9, 2019

\begin{abstract}
Current research indicates that pediatric patients experience quality-of-life (QOL) issues during recovery from concussion. However, measures of health related QOL issues in youth have generally focused on chronic illness and do not address the unique experience of brain injury. The Pediatric Life After Concussion Evaluation Scale (PLACES) was developed to gain a better understanding of youth perspectives on QOL during recovery from concussion. The PLACES is a self-rating instrument examining patient perspectives on cognition, emotion, social life, and school support. Study participants were 277 patients, receiving treatment for concussion at a single regional concussion clinic. Examination of reliability using Cronbach's alpha indicated strong performance with internal consistency $(\mathrm{r}=.92)$. The Post-Concussion Symptom Scale (PCSS) was used for comparison as this measure is commonly used to examine patient symptoms in clinical settings. Convergent and divergent validity of the PLACES was examined. Convergent validity was evident across the two measures $(r=.732, p=.001)$. Exploratory factor analysis identified 5 factors and indicated divergent factors related to emotion, social concerns, and school. Analyses demonstrated that the PLACES domains measure different aspects of quality of life issues during recovery from concussion than traditional symptom scales, thereby offering support for the proposed domain structure of the PLACES. This measure appears to provide additional information that is clinically relevant and not available on symptom scales. Initial psychometrics indicate that the PLACES has the potential to function as a QOL measure specific to concussion and will assist in greater specificity of treatment during recovery.
\end{abstract}

Keywords: Concussion, Mild Traumatic Brain Injury, Health-Related Quality of Life, Recovery, School

\section{Introduction}

Emerging research indicates that for many individuals who sustain a concussion there is an impact on quality of life (QOL) during recovery and possibly after initial symptoms abate. For students, a concussion injury can be disruptive to learning and functioning in school settings [1-3]. The impact of a head injury on a wide range of domains of function leads to a greater likelihood for QOL issues for the injured child [4-7].

Although physical symptoms are the most apparent in the immediate period after concussion, symptoms related to cognition and mood emerge more gradually [8-9]. It is not unusual for patients with concussion to experience negative emotions during the recovery period [8, 10-11]. In addition, secondary symptoms related to frustration, emotional response, and regulation of executive functions may also 
occur $[8,10]$.

Although physical symptoms resolve within 30 days for most children and adolescents with concussion, factors related to QOL, such as behavior, cognition, and emotions, may linger for several months [12-15] and affect up to $50 \%$ of individuals [15]. For a portion of injured children and adolescents, symptoms will linger beyond 30 days $[2,7,9$, 16-19]. Nevertheless, even children who report full recovery from symptoms report lower QOL related to school functioning for 12 weeks after injury7. In turn, lingering symptoms affect quality of life of children and adolescents with concussion $[5,7,20]$.

Health related QOL involves the interaction of multiple dimensions of function to form an overall picture of health, including physical symptoms, physical, emotional, and social functioning $[4,20,21]$. The construct of health related QOL differs from QOL because there is a focus on health status as part of the evaluation of QOL [20]. Symptoms related to concussion are experienced across multiple domains and impact functioning $[5,7,22]$. For example, children with persistent symptoms after concussion report lower health related QOL across physical, emotional, social, and school domains7.

A complicating factor when considering health related QOL issues is that QOL after concussion may affect functioning across multiple domains and varies over time $[4$, $7,21]$. Perceptions and concerns by parents and physicians may change as some symptoms linger and others abate [4]. In addition, ratings of the domains that are measured as part of QOL often change depending on the stage and pace of recovery [4]. For example, school functioning is the last domain to improve for children after concussion [3, 7]. Gathering information about perceptions of QOL during recovery is an important aspect of treatment and should be routinely included [22-24].

Students suffering from concussion require close monitoring to measure progress, minimize symptoms, and reduce stress from learning $[25,26]$. Further, the pace and complexity of recovery profiles indicates that each child and adolescent requires an individualized approach [3, 18, 22]. Some pediatric patients will be able to quickly increase school demands while others will require a slower controlled process [27]. Insight into how an individual with concussion perceives his or her recovery and symptom expression may help caregivers, school staff, and medical providers make decisions about the pace of increasing demands in school.

Although it is evident that there are subjective health outcomes that result from lingering concussion symptoms, research capturing the subjective experience of pediatric concussion is limited [6, 21]. Many of the problems and deficits that linger are subtle and may not be obvious without careful investigation [19]. As a result, assessment of the pediatric patient with concussion needs to evaluate multiple modalities, such as vestibular function, cognition, and the patient's perception of his or her QOL as related to the head injury [20]. Understanding the child or adolescent's perspectives on his or her recovery is important for treatment planning $[20,21,28]$. In fact, Howell et al (2019) suggest that the level of quality of life after a concussion may indicate the need for further assessment and specialized treatment.

\subsection{Quality of Life Surveys}

QOL and pediatric patient perspectives about concussion recovery have received little attention in the literature $[6,18$, 20]. Health-related QOL research has focused primarily on chronic illness such as cancer and diabetes. Children with chronic conditions demonstrate problems with function across life domains, including emotional, social, and school domains $[28,29]$. Many QOL measures have utilized parent proxies as described above; however, children are reliable reporters and their perspectives on their illnesses are important [29]. Understanding the pediatric patient's perspective is important for developing effective treatment plans $[6,28,29]$.

One of the pediatric QOL measures used most commonly in the literature is the Pediatric Quality of Life Inventory (PedsQL). This instrument assesses some aspects of the physical, emotional, social, and school functioning [30]. Generally, the PedsQL has shown good validity and reliability, but the school functioning domain has not demonstrated consistent validity and reliability across ages $[29,30]$. This may be a result of the different demands that schools place on students at the various levels of education. Although generally reliable, the PedsQL does not address the unique concerns that pediatric patients may have related to the specific impact of a concussion on QOL domains.

\subsection{Purpose of This Study}

The Pediatric Life After Concussion Evaluation Scale (PLACES) was developed to gain a better understanding of youth perspectives on their quality of life during recovery from concussion. The instrument was developed in order to capture the unique experiences and perspectives of youth with concussion injury. This study reports the results of preliminary evaluation of the properties of the PLACES and examines whether the measure may provide additional information not captured by symptom scales that are typically used to track recovery.

\section{Method}

\subsection{Participants}

Study participants were 277 patients receiving treatment for concussion at a single regional concussion clinic. Data were collected from study participants in October and November 2016 at the participant's medical appointment. Inclusion criteria for this study were as follows: (a) concussion was verified and diagnosed clinically during physical examination by the physician at the concussion clinic who has specific expertise and training in concussion and sports medicine. Patients were diagnosed through the clinic's standard concussion evaluation including 
comprehensive history, physical examination and symptom ratings. Concussion diagnosis was based upon factors including mechanism of injury, elevated symptom burden on PCSS, examination demonstrating deficits below the normal range in one or more of the following domains: vestibular, oculomotor, and balance, and review of SCAT5 or ImPACT, when available, demonstrating change from baseline for athletes or scores far below expectation for non-athletes; (b) participants were students in elementary school to college; (c) participants were able to follow the directions; , and (d) participants completed both rating scales. Participants were excluded if they were not between the age ranges of 9 to 21 . The rating scales were part of the participant's regular medical chart. Institutional Review Board approval was granted from both the university and hospital.

Previous research showed that the typical demographics at the data collection site consists of a clinical sample with approximately $8 \%$ of patients with school supports including 504 and IEP plans [33]. Approximately $85 \%$ of patients sustained concussion while engaged in sports with approximately $15 \%$ of patients with concussion sustained injury from causes such as falls and accidents [33].

\subsection{Measures}

\subsubsection{Pediatric Life After Concussion Evaluation Scale}

The Pediatric Life after Concussion (PLACES), a pencil and paper self-rating instrument, was developed to examine pediatric concussion patients' self-perceptions of their recovery from concussion. Research has indicated that children are reliable reporters and their perspectives on their illnesses are important [29, 34]. Measures such as the Pediatric Quality of Life Inventory (PedsQL) were developed to address quality of life during chronic and acute health issues such as pediatric diabetes. Generally, the PedsQL has shown good validity and reliability, but the school functioning domain has not demonstrated consistent validity and reliability [29]. Furthermore, questions concerning issues such as lack of friends, inability to lift objects, and missing school for hospital visits did not consistently apply to the challenges faced during concussion recovery. As a result, the research team used the PedsQL as a model but created questions more relevant to concussion recovery.

The PLACES assessment used in this study emerged from a preliminary study that was performed to determine whether pediatric patients could provide information about quality of life issues related to concussion. The measure was developed by examining pediatric health related quality of life measures and in consultation with clinical and school professions. The questions were presented to a group of neuropsychologists, medical professionals, and students and adjustments were made based upon recommendations. The measure was then piloted. The preliminary study participants were 118 patients with concussion, age $10-18$, receiving treatment at a regional sports medicine concussion clinic. The preliminary study showed that pediatric patients identified concerns across domains, including mood, thinking, frustration with symptoms, and social relationships [31, 32]. Preliminary analyses concerning psychometric properties indicated that the instrument developed to examine pediatric patient perspectives demonstrated good reliability for the total scale and the constructed factors. Cronbach's alpha for the scale as a whole was.941, and Cronbach's alpha for the subscales ranging from.83-.93 [31, 32].

As a result of the preliminary study, changes were made to the PLACES measure. The items of the measure were grouped into the four categories based on principal component factor analysis and face validity in order to improve clarity of the measure. In addition, an example for completing the scale was added, headings were added for each section, and wording was clarified for two items.

The PLACES measure used in this study consists of 20 questions with a 4 th-5th grade reading level. The instrument typically takes less than $15 \mathrm{~min}$ to complete. There are two sections in this instrument. Demographic questions regarding the participant's gender, age, number of weeks since the injury, and perception of recovery status were grouped in Section A. Perception of recovery status was determined by participants rating how recovered they believed they were using an estimated "percent recovered" number. No specific identifying information was gathered on the instrument.

Twenty perception questions related to participant's experiences during recovery from concussion were grouped in Section B. The scale uses a 4-point Likert rating for each item; 0 ("Not at all"), 1 ("A little true"), 2 ("Mostly true"), and 3 ("Very true"). The questions were designed to tap the subjective experience of youth with concussion not captured by existing self-rating measures. Questions were grouped in four domains impacted by concussion and included, school support, social experiences, emotional status, and cognition. The School Support domain includes 5 questions that address

teachers' understanding of recovery needs, level of support provided by teachers and school staff, availability of accommodations, use of available accommodations, and perception of the relationship between school demands and recovery.. The Social domain includes 5 questions that address the impact of concussion on participation in social activities, the impact on time spent with peers, desire to be with friends, the impact on relationships with peers, and whether participants felt left out because symptoms interfere with social interaction. The Emotion domain includes 5 questions that address perceptions of mood, frustration with symptoms, feeling overwhelmed, worry about recovery, and life disruption. The Cognition domain includes 5 questions that address perceptions of impact of symptoms on his or her cognition, memory and learning, attention, and feelings of difficulty and impairment in school.

\subsubsection{Post-Concussion Symptom Scale}

The Post Concussion Symptom Scale (PCSS) is a measure designed to capture the state of symptoms at the time of its completion $[34,35]$. The measure was originally designed for use with adults but has been validated for children as young as 5 years old $[14,34]$. The instrument has also been validated using both neuropsychological measures and scans, 
such as functional magnetic resonance imaging (fMRI) [36]. The PCSS was used to help examine the validity of the PLACES as this measure is commonly used in clinical settings and was the primary measure of symptom expression at the data collection site. The PCSS is a list of 22 symptoms described in single words or short phrases that has a fourth grade reading level. The scale addresses physical/somatic, cognitive, and emotion symptoms. The PCSS uses a 7-point Likert scale ranging from "Not at All" to "Very True." The directions ask the individual to rate his or her symptoms on the day of administration. The ratings for all 22 symptoms are then summed to produce a total concussion symptom. The higher the score, the greater the symptom load.

\subsection{Procedure}

When the participants checked in for their appointment at the concussion clinic, they were provided with a package of questionnaires and instructed to complete the measures in the waiting room. The PLACES and the PCSS were completed by participants as part of the package of symptom questionnaires that all patients completed at their medical visits at the regional concussion clinic. Participants were encouraged to complete the measures independently and to ask clinic staff if they had questions. Measures were read to participants by clinic staff if the participant indicated that symptom exacerbation occurred when reading. The staff at the regional concussion clinic collected the measures from the participants, deidentified forms, and entered PLACES and PCSS responses into a database that was provided to the research team.

\subsection{Obtaining Raw Scores for the PLACES and the PCSS}

PLACES Scores. Social, Emotion, and Cognition Composite scores were calculated for each domain by summing the scores for the five questions in each domain. For these three domains, scores represent the degree of perceived agreement with statements about impairment, with low scores indicating little or no perceived impairment and high scores indicating a lot of perceived impairment. A School Support domain score was obtained by summing four of the five questions related to supports provided by the students' schools. For this domain, scores represent perceptions of the degree of support provided, with low scores indicating little or no perceived support and high scores indicating a lot of perceived support. A total PLACES score was calculated by summing all of the questions in the cognition, emotion, and social domains, plus the one symptom question in the school domain. The PLACES Total score represents the extent of perceptions of impairment overall, with low scores indicating little or no perceived impairment overall and high scores indicating a lot of perceived impairment overall.

PCSS Scores. The total PCSS score was calculated by adding responses to all 22 questions. The PCSS total score represents a combination of the numbers of symptoms reported and the severity of these symptoms; the higher the score the greater the number of symptoms reported and/or the greater the severity of the symptoms reported. Additionally, PCSS Cognition, Emotion and Physical Subscales were constructed for the purposes of comparison with the PLACES domain scores. Four items that address cognitive symptoms (difficulty concentrating, difficulty remembering, feeling foggy, feeling slow) were summed to produce the Cognitive Subscale score. Four items that address emotion symptoms (irritability, sadness, nervousness, more emotional) were summed to produce the Emotion Subscale. The remaining 14 items that address physical and somatic symptoms were combined to produce the Physical Subscale.

\subsection{Sample Demographics}

The age of participants ranged from 9 to $21(\mathrm{M}=14.83$, $\mathrm{s}=2.3)$. The majority of participants were between ages 13 and $17(75.5 \%)$, with lower percentages of younger children (age 9-12, 16.5\%) and older adolescents (age 18$21,7.9 \%)$. There were slightly more male $(52.3 \%)$ than female participants $(47.7 \%)$. Time since injury ranged from 1 week to 90 weeks $(M=9.3, \mathrm{~s}=7.7)$. The majority of participants attended school for full days (71.8\%). Students attending school for partial days accounted for $16.2 \%$ of participants. One student received in-home instruction and $10.8 \%$ had not yet returned to school after their injury.

\section{Results}

Subject's responses to the PLACES and PCSS were analyzed to establish the psychometric properties of the PLACES Domain and Total scores.

\subsection{Sample Response Characteristics}

Raw score distributions were generated for the PLACES Domain and Total scores and the PCSS Subscale and Total scores and measures of central tendency and variability were calculated. Table 1 shows the means, standard deviations, and skewness values that were obtained.

\subsection{Reliability}

Internal consistency reliability estimates for the PLACES Total and Domain scores and the PCSS Total and Subscale scores were computed using Cronbach's coefficient alpha. The internal consistency estimates for the PLACES composite scores were as follows: PLACES Total score $r$ $=.92$; Cognition $r=.93$, Emotion $r=.89$, Social $r=.85$, and School Support $r=.74$. The internal consistency estimates for the PCSS total and Subscale scores were as follows: Total score $r=.94$; Cognition $r=.89$, Emotion $r=.87$, and Physical $\mathrm{r}=.90$

Prior analyses using this measure demonstrated skewness and collinearity within acceptable limits [37]. 
Table 1. Means, Standard Deviations and Skewness values for the PLACES Composite Scores and the PCSS Scale and Subscale Scores.

\begin{tabular}{lllll}
\hline Domain & Mean & Standard Deviation & Max Score & Skewness \\
\hline PLACES Total & 17.17 & 12.29 & 48 & .404 \\
PLACES Cognition & 5.06 & 4.74 & 15 & .695 \\
PLACES Emotion & 5.79 & 4.73 & 15 & .465 \\
PLACES Social & 5.31 & 4.17 & 15 & .634 \\
PLACES School & 9.07 & 3.16 & 132 & -1.216 \\
PCSS Total & 20.24 & 22.28 & 84 & 1.405 \\
PCSS Physical & 13.32 & 14.06 & 24 & 1.198 \\
PCSS Cognition & 4.82 & 5.88 & 24 & 1.354 \\
PCSS Emotion & 2.92 & 5.01 & 2.042 \\
\hline
\end{tabular}

Note: PLACES is the acronym for the Pediatric Life After Concussion Evaluation Scale and PCSS is the Post Concussion Symptom Scale

\subsection{Age}

In order to examine whether age was a significant variable to be accounted for analyses were conducted. There was only one low but significant correlation between age and PLACES Emotion domain $(r=.148, p=.05)$. Otherwise, there were no significant correlations between age and any of the other domains. In previous research37 the sample was divided by age into three groups: group 1 consisted of participants aged 9-12 years (16.2\% of sample), group 2 was aged 13-17 (75.5\%), and group 3 was aged 18-21 (8.3\%). The KruskalWallis one-way analysis of variance was used to test for significance of age differences for the PLACES Total and domain score and the PCSS Total and Subscale scores. The results showed no significant differences between the age groups for either concussion symptoms or quality of life ratings, indicating that both measures perform similarly across age groups.

\subsection{Correlations}

Correlations were calculated to examine relationships among PLACES and PCSS scores, post-concussive symptoms, age, and perception of percent recovered (Table 2). A strong positive relationship between the total PLACES and total PCSS scores $(\mathrm{r}=.732, \mathrm{p}=.001)$ was found, offering evidence of convergent validity for the two total scale scores. Elevated post-concussion symptom load on the PCSS was correlated with worse QOL (total PLACES). Further, total post-concussion symptoms, as measured by the PCSS, was correlated strongly with the QOL domains of the PLACES (Cognition $r=.73, p<.01$; Emotion $r$
$=.63, p<.01$ ); Social $r=.55, p<.01)$. No significant relationship was found between perception of school support and postconcussion symptoms $(r=-.04, p<.54)$.

Total PLACES had a strong negative correlation with perception of percent recovered $(r=-.55, p<.01)$. Similarly, there was a strong negative correlation between rating of percent recovered and total symptoms on the PCSS $(r=-.60$, $p<.01)$. A lower symptom load was correlated with a

rating of percent recovered and total symptoms on the PCSS $(r=-.60, p<.01)$. A lower symptom load was correlated with a greater sense of recovery. A strong negative correlation was found between perception of percent recovered and the PLACES Cognition domain score $(r=$ $.55, p<.01)$. Moderate negative correlations were found between percent recovered and PLACES Emotion $(r=-.40, p$ $<.01)$ and Social $(r=-.44, p<.01)$ domain scores. The more recovered the individual felt, the lower the symptom scores related to cognition, emotion, and social QOL.

The PCSS Cognition Subscale had a strong positive correlation with the PLACES Cognition domain score $(r=-.78$, $p<.01)$ suggesting that the items of these two scales are measuring a similar construct. Similarly, the PCSS Emotion Subscale and the PLACES Emotion domain scores were strongly correlated $(r=-.62, p<.01)$. The PLACES Total score was strongly correlated with both the PCSS Cognition Subscale $(r=-.70, p<.01)$ and the PCSS Emotion Subscale $(r=-.59, p$ $<.01)$. The correlational analysis offers evidence of both convergent and divergent validity when comparing the PLACES scores with the PCSS scores, post-concussive symptoms, and age.

Table 2. Correlations Among PLACES Domains, PCSS, and Injury Demographics.

\begin{tabular}{|c|c|c|c|c|c|c|c|c|c|c|}
\hline & PLACES Cognition & $\begin{array}{l}\text { PLACES } \\
\text { Social }\end{array}$ & $\begin{array}{l}\text { PLACES } \\
\text { Emotion }\end{array}$ & $\begin{array}{l}\text { PLACES } \\
\text { School }\end{array}$ & $\begin{array}{l}\text { PLACES } \\
\text { Total }\end{array}$ & $\begin{array}{l}\text { PCSS } \\
\text { Total }\end{array}$ & $\begin{array}{l}\text { PCSS } \\
\text { Cog }\end{array}$ & $\begin{array}{l}\text { PCSS } \\
\text { Emot }\end{array}$ & $\begin{array}{l}\text { \% } \\
\text { Recovered }\end{array}$ & Age \\
\hline Cognition & 1 & $.561 * *$ & $.714 * *$ & .002 & $.870 * *$ & $.733 * *$ & $.776^{* *}$ & $.525^{* *}$ & $-.547 * *$ & .076 \\
\hline Social & & 1 & $.685 * *$ & $.161^{*}$ & $.837^{* *}$ & $.553 * *$ & $.455 * *$ & $.455^{* *}$ & $-.439 * *$ & -.008 \\
\hline Emotion & & & 1 & .029 & $.913 * *$ & $.629 * *$ & $.595 * *$ & $.619^{* *}$ & $-.404 * *$ & $.148^{*}$ \\
\hline School & & & & 1 & .066 & -.040 & -.065 & -.069 & -.017 & .009 \\
\hline PLACESTot & & & & & 1 & $.732 * *$ & $.704 * *$ & $.594 * *$ & $-.552 * *$ & .096 \\
\hline PCSS Tot & & & & & & 1 & $.896 * *$ & $.798 * *$ & $-.597 * *$ & .057 \\
\hline PCSS Cog & & & & & & & 1 & $.695 * *$ & $-.535 * *$ & .024 \\
\hline PCSS Emo & & & & & & & & 1 & $-.297 * *$ & -.057 \\
\hline$\%$ Recover & & & & & & & & & 1 & $.189 * *$ \\
\hline Age & & & & & & & & & & 1 \\
\hline
\end{tabular}




\subsection{Exploratory Factor Analysis}

The 20 PLACES items and the PCSS items of the Cognition and Emotion Subscales were factor analyzed using exploratory principal component analysis with Varimax rotation. The analysis yielded 5 factors explaining $71.3 \%$ of the total variance (Table 3). A minimum value of.4 was established for the rotation. Kaiser-Meyer-Olkin measure of sampling adequacy was.928 and Bartlett's test of Sphericity had a significance of.000, indicating that the items were adequately related and appropriate for factor analysis.

The first factor is labeled Cognition as the 5 items of the PLACES Cognition domain and the 4 items of the PCSS
Cognition subscale all loaded highly on this factor. This first factor accounted for $20.85 \%$ of the variance. The highest loadings on the third factor were for the 5 items of the PLACES Social domain. This factor accounted for $14.03 \%$ of the variance. The fourth factor consisted of PLACES items related to the feelings of frustration and being overwhelmed and was named Emotional Impact accounting for $10.7 \%$ of variance. This factor is distinct from the Mood factor upon which the PCSS emotion items related to emotion all load. The fifth factor consisted exclusively of high loadings for 4 of the 5 items of the PLACES School domain, accounting for $10.66 \%$ of the variance.

Table 3. Factor Analysis Table for Quality of Life During Recovery from Concussion.

\begin{tabular}{|c|c|c|c|c|c|}
\hline Item & Cognition & Mood & Social & Emotional Impact & School \\
\hline PLACES Symptoms make it hard to think & .769 & & & & \\
\hline PLACES Symptoms make it hard to pay attn & .807 & & & & \\
\hline PLACES Trouble learning new information & .758 & & & & \\
\hline PLACES Trouble remembering information & .794 & & & & \\
\hline PLACES Symptoms impair me in school & .694 & & .468 & & \\
\hline PCSS Feeling foggy & .674 & .558 & & & \\
\hline PCSS Difficulty remembering & .724 & & & & \\
\hline PCSS Feeling slowed down & .549 & .574 & & & \\
\hline PLACES Concussion has disrupted my life & & & .467 & .656 & \\
\hline PLACES I feel frustrated by symptoms & & & .412 & .664 & \\
\hline PLACES My symptoms effect how I feel & & .444 & & .648 & \\
\hline PLACES I feel overwhelmed & & & & .686 & \\
\hline PCSS More emotional & & .812 & & & \\
\hline PCSS Nervousness & & .692 & & & \\
\hline PCSS Irritability & & .777 & & & \\
\hline PCSS Sadness & & .815 & & & \\
\hline PLACES Prevented from time w/friends & & & .783 & & \\
\hline PLACES Prevented participation activities & & & .517 & & \\
\hline PLACES I feel left out because of symptoms & & & .685 & .401 & \\
\hline PLACES Less social since injury & & & .746 & & \\
\hline PLACES Symptoms effect way I interact & & & .725 & & \\
\hline PLACES School demands - recovery longer & & & .423 & & \\
\hline PLACES Teachers are understanding & & & & & .884 \\
\hline PLACES School has been helpful & & & & & .873 \\
\hline PLACES School provides accommodations & & & & & .903 \\
\hline PLACES I use my accommodations & & & & & .725 \\
\hline
\end{tabular}

\section{Discussion}

The purpose of this study was to examine whether a quality of life measure designed specifically for concussion could provide useful information beyond that provided by symptom scales. The Pediatric Life After Concussion Evaluation Scale (PLACES) was developed to explore youth patient perspectives about recovery from concussion [31, 32].

The PLACES consists of a demographic section and 20 questions in four domains, Cognition, Social, Emotion, and School Support and provides scores for each domain and a Total score that sums all item scores. The Total score and each of the domains demonstrate adequate internal consistency. Previous analyses established that there were no age differences evident in response patterns suggesting that this measure produces similar results across the age span from age 9 to 21 .

The PCSS is a measure that has demonstrated reliability and validity for pediatric populations [14, 34-36], and so was used as an anchor for comparison with the PLACES. There was a strong positive correlation between the PCSS and the PLACES total scores indicating convergent validity. This is an expected finding as both measures address symptoms associated with concussion. Similarly, the domains of cognition and emotion on the PLACES and PCSS are strongly correlated, indicating that the items are related and likely measure similar constructs. The Social domain was not as highly correlated with the cognitive and emotion domains, 
supporting the contention that the social domain items assess an important dimension apart from what is assessed with the items of the other domains. The School Support domain demonstrated divergence from the cognitive, emotion and social scales as anticipated. The correlations among the PLACES and PCSS scores reflected patterns of convergent and divergent relationships that help to establish the validity of the PLACES instrument as a post-concussion measure of quality of life for students. The PLACES expands upon symptoms captured in the PCSS and includes areas related to quality of life during recovery that are not captured by the PCSS.

Factor analysis of the PLACES items and the PCSS items related to cognition and emotion identified five factors. The first factor consisted of items from the PLACES and PCSS that assessed symptoms and concerns about cognition, memory, and attention. The second factor generally consisted of the PCSS items related to mood, such as irritability, sadness, and increased emotionality. This factor was distinct from the fourth factor which consisted of PLACES items from the Emotion domain that related to the emotional impact of concussion, such as feelings of frustration, being overwhelmed, and feeling that the concussion has disrupted one's life. The PLACES Social domain items loaded on the third factor, and the PLACES School Support items loaded on the fifth factor. The factor analysis shows that the PLACES domains hold together and measure different aspects of quality of life issues during recovery from concussion thereby offering support for the proposed domain structure of the PLACES. In addition, this provides support for the idea that youth recovering from concussion would benefit from questions concerning social life, frustration, and school support [8, 10, 11, 20, 21].

This study has clinical implications for youth who have sustained concussion. Children and adolescents will benefit from the opportunity to rate their concerns during recovery from concussion. Medical professionals, clinicians, and school personnel will be able to provide more individualized and effective treatment plans when they understand the youth's concerns and challenges during the recovery period [6, 28, 29].

\section{Limitations}

While this study does offer support for the reliability and validity of the PLACES, the conclusions may be limited by the characteristics of the participants included in the sample. The participants were patients seeking treatment from a specialized concussion clinic, and so the severity and intensity of symptoms may be elevated compared to individuals treated in more general medical settings. The data set did not include pre-injury diagnoses or previous concussions and so prior learning, mental health factors, and health history were not considered. In addition, the clinic provided data from all patients seen within a 2-month period. It is estimated that a small portion of participant responses were from patients who were seen at two different points in their recovery, based on the typical follow-up schedule at the clinic. Although the repeat visits were part of the routine protocol of the clinic, it is possible that there was a unique commonality among these patients. While the number of participants is adequate for a preliminary assessment of the psychometric properties, the planned large scale study of the measure will help to further verify the reliability and validity of the instrument.

\section{Conclusions}

Quality of life is emerging as an important area of study and clinical practice with youth who have sustained a concussion [4-7]. Addressing the youth's perspectives on his or her recovery from concussion is important for treatment planning and progress monitoring [20, 21, 24, 28]. The Pediatric Life After Concussion Evaluation Scale (PLACES) was developed in order to capture the unique experiences and perspectives of individuals with concussion injury.

The PLACES consists of a demographic section and 20 questions in four domains, Cognition (Cognition), Social, Emotion, and School Support. The instrument has demonstrated good internal reliability and consistency. Content validity was supported during the development of the measure. The current statistical analyses indicated that the PLACES demonstrates a pattern of relationships with the PCSS that are consistent with expectations, thereby supporting the validity of the PLACES as a post-concussion measure. Further, an exploratory factor analysis showed that the PLACES domains measure distinct aspects of concussion recovery thereby supporting the proposed domain structure of the PLACES and offering further support for its validity. Overall, the initial analyses offer support for the reliability and validity of the PLACES domain and Total scores. Initial indications are that the PLACES has the potential to function as a quality of life measure specific to concussion that will assist greater specificity in treatment and progress monitoring. The PLACES has the potential to help clinicians and schools to understand better individual student's needs during recovery from concussion.

\section{References}

[1] Burke, M. J., Fralick, M., Nejatbakhsh, N., Tartaglia, M C., \& Tator, C. H. (2015). In search of evidence-based treatment for concussion: Characteristics of current clinical trials. Brain Injury, 29 (3), 300-305.

[2] Purcell, L., Harvey, J., \& Seabrook, J. A. (2016). Patterns of recovery following sport-related concussion in children and adolescents. Clinical Pediatrics, 55 (5), 452-458.

[3] Sady., M. D., Vaughan, C. G., \& Gioia, G. E. (2011). School and the concussed youth: Recommendations for concussion education and management. Physical Medicine and Rehabilitation Clinics of North America, 22, 701-719.

[4] DiBattista, A., Soo, C., Catroppa, C., \& Anderson, V. (2012). Quality of life in children and adolescents post-TBI: A systematic review and meta-analysis. Journal of Neurotrauma, 29: 171-1727. 
[5] Houston M. N., Bay R. C., McLeod T. C. V. (2016) The relationship between post-injury measures of cognition, balance, symptom reports and health-related quality-of-life in adolescent athletes with concussion. Brain Injury. 30: 891898.

[6] Moran, L. M., Taylor, H. G., Rusin, J., Bangert, B., Dietrich, A., Nuss, K. E., Wright, M., Minich, N., \& Yeates, K. O. (2011). Quality of life in pediatric mild traumatic brain injury and it's relationship to post concussive symptoms. Journal of Pediatric Psychology, 37 (7), 736-744.

[7] Novak, Z., Aglipay, M., Barrowman, N., Yeates, K. O., Beauchamp, M. H., Gravel, J., ... Novak, R. L. (2016). Association of persistent postconcussion symptoms with pediatric quality of life. JAMA Pediatrics, 170 (12), E1-E8.

[8] Eisenberg, M., Meehan, W., \& Mannix, R. (2014). Duration and course of post-concussive symptoms. Pediatrics, 133 (6), 999-1006.

[9] Heyer, G. L., Schaffer, C. E., Rose, S. C., Young, J. A., McNally, K. A., \& Fischer, A. N. (2016). Specific factors influence postconcussion symptom duration among youth referred to a sports concussion clinic. The Journal of Pediatrics, 174: 33-8.

[10] Chaput, G., Giguere, J.-F., Chauny, J.-M., Denis, R., \& Lavigne, G. (2009). RelationshipAmong subjective sleep complaints, headaches, and mood alterations following a Mild traumatic brain injury. Sleep Medicine, 10 (2009), 713-716.

[11] Luis, C., \& Mittenberg, W. (2002). Mood and anxiety disorders following pediatric traumatic brain injury: A prospective study. Journal of Clinical and Experimental Neuropsychology, 24 (3), 270-279.

[12] McCrory, P., Meeuwisse, W., Johnston, K., Dvorak, J., Aubrey, M., Molloy, M., \& Cantu, R. (2009). Consensus statement on concussion in sport: the 3rd International Conference on Concussion in Sport held in Zurich, November 2008. Journal of Science and Medicine in Sport, 12, 340-351.

[13] National Center for Injury Prevention and Control. (2007). Facts for Physicians, HeadsUp: Brain Injury in Your Practice. Atlanta, GA: Centers for Disease Control and Prevention.

[14] Zemek, R. L., Farion, K. J., Sampson, M., \& McGahern, C. (2013). Prognosticators of persistent symptoms following pediatric concussion. Journal of the American Medical Association/Pediatrics, 167 (3), 259-265.

[15] Crowe, L., Collie, A., Hearps, S., Dooley, J., Clausen, H., Maddocks, D., McCrory, P., ...Anderson, V. (2016). Cognitive and physical symptoms of concussive injury in children: A detailed longitudinal recovery study. British Journal of Sports Medicine, 50, 311-316.

[16] Field, M., Collins, M. W., Lovell, M. R., \& Maroon, J. (2003). Does age play a role in recovery from sports related concussion: A comparison of high school and collegiate athletes. The Journal of Pediatrics, 142, 546-553.

[17] Harmon, K. G., Drezner, J. A., Gammons, M., Guskiewicz, K. M., Halstead, M., Herring, S. A., ... Roberts, W. O. (2013). American medical society for sports medicine position statement: Concussion in sport. British Journal of Sports Medicine, 47, 15-26.

[18] Makdissi, M., Davis, G., Jordan, B., Patricios, J, Purcell, L., \& Putikian, M. (2013). Revisiting the modifiers: How should the evaluation and management of acute concussions differ in specific groups? British Journal of Sports Medicine, 47, 314320 .

[19] Raikes, A. C. \& Smart, J. (2015). The effects of sport-related concussions sustained during childhood and adolescence, and the need for educational accommodation. Current research Concussion, 2 (1), 25-31.

[20] McLeod, T. C., Houston, M. N., \& Welch, C. E. (2015). A pediatric perspective on sport-related concussion. Kinesiology Review, 4, 131-155.

[21] Fineblit, S., Selci, E., Loewen, H., Ellis, M., \& Russell, K. (2016). Health related quality of life after pediatric mild traumatic brain injury/concussion: A systematic review. Journal of Neurotrauma, 33, 1561-1568.

[22] McLeod T. C. V, Wagner A. J., Bacon C. E. W. (2017) Lived experiences of adolescent athletes following sport-related concussion. Orthop Journal of Sports Medicine. 2017; 5: 2.

[23] Pieper, P. \& Garvan, C. (2014). Health-related quality-of-life in the first year following achildhood concussion. Brain Injury, 28 (1), 105-113.

[24] Howell, D. R., Wilson, J. C., Kirkwood, M. W., \& Grubenhoff, J. A. (2019). Quality of life and symptom burden 1 month after concussion in children and adolescents. Clinical Pediatrics, 58 (1), 42-49.

[25] Halstead, M. E., McAvoy, K., Devore, C. D., Carl, R., Lee, M., \& Logan, K. (2013). Returning to learning following a concussion. Pediatrics, 2013, 948-957.

[26] Popoli, D., Burns, T., Meehan, W., \& Reisner, A. (2014). CHOA concussion consensus establishing a uniform policy for academic accommodations. Clinical Pediatrics, 53 (2), 217224.

[27] Master, C. L., Gioia, G. A., Leddy, J. J, \& Grady, M. F. (2012). Importance of 'return-to-learn' in pediatric and adolescent concussion. Pediatric Annals, 41 (9), 1-6.

[28] Testa M. A. \& Simonson D. C. Assessment of quality-of-life outcomes. (1996) New England Journal of Medicine. 334: $835-840$.

[29] Varni, J. W., Limbers, C., \& Burwinkle, T. M. (2007). Literature review: Health-related quality of life measurement in pediatric oncology: Hearing the voices of the children. Journal of Pediatric Psychology, 32 (9), 1151-1163.

[30] Varni, J. W., Seid, M. M., \& Kurtin, P. S. (2001). PedsqL 4.0: Reliability and validity of the pediatric quality of life inventory version 4.0 generic core scales in healthy and patient populations. Medical Care, 39 (8), 800-812.

[31] Clair, R. M., Tresco, K., Goodman, A., Weinberger, I., McCloskey, G., \& Allen, S. L. (2016, June). Evaluating quality of life after concussion: The psychometric properties of a recovery profile. Poster session presented at the meeting of the American Academy of Clinical Neuropsychology, Chicago, IL

[32] Clair, R., Henigan, M., Tresco, K., Goodman, A., Weinberger, I., \& Levin Allen, S. (2016, May). The subjective experience of mild traumatic brain injury in pediatric patients. Poster Session presented at the $9^{\text {th }}$ World Congress of Neurorehabilitation, Philadelphia. 
[33] Gerrald, L., Reott, Goodman, A., Levin Allen, S. \& Clair, R. (2018). The Relationship Between Preexisting Conditions and Recovery Patterns in Pediatric Concussion Patients. Poster Session presented at International Neuropsychological Society, $46^{\text {th }}$ Meeting, Washington DC.

[34] Lovell, M. R., Iverson, G. L., Collins, M. W., Podell, K., Johnston, K. M., Pardini, D., ... Maroon, J. (2006). Measurement of symptoms following sports-related concussion: Reliability and normative data for the postconcussion scale. Applied Neuropsychology, 13 (3), 166-174.

[35] Kontos, A. P., Elbin, R. J., Schatz, P., Covassin, T., Henry, L., Pardini, J., \& Collins, M. W. (2012). A revised factor structure for the post-concussion symptom scale: Baseline and postconcussion factors. American Journal of Sports Medicine, 40 (10), 2375-2384.

[36] Chen, J.-K., Johnston, K. M., Collie, A., McCrory, P., \& Ptito, A. (2007). A validation of the post concussion symptom scale in the assessment of complex concussion using cognitive testing and functional MRI. Journal of Neurology, Neurosurgery, and Psychiatry, 78, 1231-1238.

[37] Clair, R., Levin Allen, S., Goodman, A., \& McCloskey, G (2019). Gender differences in quality of life during recovery concussion. Applied Neuropsychology: Child, DOI: 10.1080/21622965.2018.1556102. 